\title{
Análise da intensidade dos serviços de cuidado prestados aos Utentes Idosos do Serviço de Apoio Domiciliário
}

\author{
Analysis of the intensity of care provided to Elderly Users of the Home Support Service
}

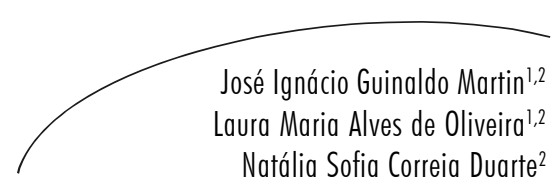

Resumo

\begin{abstract}
A intensidade dos cuidados prestados pelo Serviço de Apoio Domiciliário (SAD) é um tema importante em diversos países. Em alguns casos, é considerada um critério de qualidade e a base de cálculo do pagamento do Serviço de Apoio Domiciliário. Este estudo tem como objectivos analisar quantitativamente a intensidade das tarefas de cuidado prestadas no Serviço de Apoio Domiciliário em Portugal, em termos de periodicidade e tempo despendido (em minutos), e fazer o cálculo dos valores de referência do consumo de tempo para cada tarefa de cuidado nas Actividades de Vida Diária (Básicas e Instrumentais). No total foram analisadas oito instituições promotoras do Serviço de Apoio Domiciliário, as quais se agruparam segundo o contexto territorial a que pertencem (rural/ urbano) e a capacidade de utentes que possuem (pequena/ grande dimensão). De cada instituição foram aleatoriamente seleccionados seis utilizadores, resultando em amostra final de 48 sujeitos. A recolha de dados foi realizada com o recurso ao Protocolo de Serviço de Apoio Domiciliário (ProSAD). Os resultados indicam que a intensidade é superior nos cuidados prestados em termos de Actividades Básicas de Vida Diária, mas os valores variam em função da presença/ausência de cuidador informal. Concluiu-se que a intensidade dos cuidados em Portugal é reduzida, sendo que os poucos serviços prestados incidem nas Actividades Básicas de Vida Diária. O tempo a ser utilizado por cada utente do Serviço de Apoio Domiciliário deve ter por base critérios objectivos, como o estado funcional do utente e a rede de suporte social.
\end{abstract}

\section{Abstract}

The intensity of care provided by Home Support Service (DSS) is a major issue in many countries. In some cases, it is considered a quality criterion and basis of calculation of payment of the Home Support Service. This study aims to quantitatively analyze the intensity of caregiving tasks provided in the Home Support Service in Portugal in terms of frequency and time spent (in minutes), and calculate

\footnotetext{
Universidade de Aveiro. Secção Autónoma de Ciências da Saúde. Campo Universitário de Santiago. Aveiro, Portugal.

2 Unidade de Investigação e Formação sobre Adultos e Idosos - UnIFai. Porto, Portugal.
}

\author{
Palavras-chave: Serviços \\ de Assistência Domiciliar. \\ Assistência Domiciliar. \\ Avaliação de Custo- \\ Efetividade. Periodicidade. \\ Distribuição Temporal. \\ Análise Quantitativa. \\ Tempo Despendido. \\ Portugal.
}


reference values of the time consumption for each task care in Activities of Daily Living (Basic and Instrumental). Eight institutions promoters of Home Support Service were analyzed, grouped according to the local context to which they belong (rural / urban) and the ability of users that have (small / large). Each institution randomly selected six users, resulting in a final sample of 48 subjects. Data collection was performed with the use of the Protocol of Home Support Service (PROSAD). The results indicate that the intensity is higher than the care provided in terms of Basic Activities of Daily Living, but the values vary depending on the presence / absence of informal caregivers. The conclusion was that the intensity of care in Portugal is reduced, and the few services focus on the Basic Activities of Daily Living. The time to be used by each user of the Home Support Service should be based on objective criteria such as the functional state of the user and the social support network.the elderly about participation in groups. Interviews were conducted with 20 people of both genders and divided into two groups, of which ten attended the Grupo Flor de Maçã, and ten attend the Grupo Melhor Idade em Ação. Data were collected through semi-structured interviews and participant observation recorded in a field diary, which occurred in places and times scheduled with supervisors and participants. The data were analyzed by the participants' speech, processed and separated into pre-established categories. It was found that participation in living groups made dramatic improvements and changes in the lives of elderly of the study and improvements in several factors can be noticed, such as health, self-esteem and recovery. Many reported that activities in groups contributed significantly to the development of the functions of daily life, besides getting a recognition and appreciation, both by families and by society in general. We conclude that participation in groups is paramount in the quest to achieve better quality of life.
Key words: Home Care Services. Home Nursing. Cost-Effectiveness Evaluation. Periodicity. Temporal Distribution. Quantitative Analysis. Portugal.

\section{INTRODUÇÃO}

São vários os estudos que se têm debruçado sobre o número de visitas aos utentes do Serviço de Apoio Domiciliário (SAD) e sobre o tempo utilizado por visita. ${ }^{1,2}$ No entanto, é muito difícil fazer comparações internacionais, uma vez que o SAD adquire características próprias nos vários países, tornando-os muito diferentes entre si, sendo uma das diferenças a intensidade do cuidado. Os EUA utilizam a intensidade dos cuidados prestados pelo SAD (periodicidade das visitas e tempo disponibilizado por tarefa) como base do cálculo para o pagamento dos serviços. ${ }^{2}$

A realidade espanhola é talvez a mais semelhante com a portuguesa, e devido às características em comum são colocados no mesmo grupo de países. ${ }^{3}$ Neste sentido, estudo realizado em Espanha ${ }^{4}$ concluiu que a duração das visitas domiciliárias tem o mínimo de 30 minutos e o máximo de três horas diárias.
Em Portugal, o SAD apresenta capacidade para 67.127 utentes, ${ }^{5}$ sendo a taxa de ocupação nacional (número de utentes/ capacidade dos SAD) de $0,92.5^{5}$

Em Portugal, a Segurança Social comparticipa de forma semelhante todas as instituições, independentemente do tipo de serviços prestados ou dos profissionais envolvidos, e a única distinção efectuada resulta do número de dias em que é prestado o cuidado, isto é, se cinco ou sete dias por semana. ${ }^{6}$

Os objectivos deste trabalho são, em primeiro lugar, descrever a intensidade de cuidado prestado em Portugal pelo SAD, e em segundo, fazer o cálculo dos valores de referência do consumo de tempo para cada tarefa de cuidado nas Actividades de Vida Diária Básicas - AVD(B) e Instrumentais - AVD(I). Estes valores são importantes porque permitem predizer o consumo de tempo necessário para um utente de acordo com a globalidade de tarefas de que necessita. Este estudo parte do princípio de que o tempo 
dispensado por tarefa varia dependendo da presença ou ausência de cuidadores informais, uma vez que estes podem influenciar o consumo de tempo da ajudante familiar domiciliária na prestação do serviço.

\section{METOdOLOGIA - Amostra e Procedimentos}

\section{Amostra das Instituições promotoras de} Serviço de Apoio Domiciliário

Para a selecção das instituições, foi utilizada uma amostragem aleatória por quotas. Controlaram-se duas variáveis (capacidade de utentes do SAD e contexto rural vs. urbano) que podem influenciar o tipo de serviços prestados. ${ }^{7}$

Obteve-se a listagem de todos os SADs do Distrito de Aveiro (126 instituições promotoras $\mathrm{SAD}$ ), assim como o número de utentes de cada instituição. ${ }^{8}$ Foi calculada a mediana do número de utentes de cada SAD, que resultou em 25 utentes. Desta forma, os SADs foram classificados em grande dimensão ( $\geq 25$ utentes) e pequena dimensão ( $\leq 25$ utentes). Os SAsD foram classificados segundo o contexto, se urbano ou rural. ${ }^{9}$

Assim, o universo foi dividido em quatro grupos: Grupo I (contexto urbano e grande dimensão) - 52 instituições; Grupo II (contexto urbano e pequena dimensão) - 65 instituições; Grupo III (contexto rural e grande dimensão) - 2 instituições; Grupo IV (contexto rural e pequena dimensão) - 7 instituições. De cada grupo, foram seleccionadas aleatoriamente duas instituições. No final, a amostra foi constituída por 8 instituições.
O protocolo foi aplicado aleatoriamente a seis utentes de cada SAD seleccionado, constituindo uma amostra total de 48 utentes de SAD.

Em todos os casos, registou-se o consentimento informado dos utentes para colaborar na recolha de dados. Desde o início do processo, foi garantida a confidencialidade da informação recolhida, seja em relação aos utentes, seja em relação às instituições de carácter social em análise, assim como a disponibilização dos resultados.

\section{Amostra dos Utentes}

A maioria dos utentes (tabela 1) é do sexo masculino $(\mathrm{n}=33 ; \%=68,8)$ e destes, mais de metade apresenta o apoio de um cuidador informal $(\mathrm{n}=19 ; \%=57,6)$. A média de idades é elevada $(\bar{X}=78,8 ; D P=9,8)$, mas na presença de cuidadores informais, a média de idades diminui $(\overline{\mathrm{X}}=76,7 ; \%=8,2)$. O número de indivíduos casados $(\mathrm{n}=18 ; \%=37,5)$ e de viúvos $(\mathrm{n}=17 ; \%=$ $35,4)$ é semelhante. A maioria apresenta níveis de educação baixos, $62,5 \%(\mathrm{n}=30)$ não completaram a escola primária (menos de 4 anos de escolaridade).

Mais de metade dos utentes de SAD apresentam a incapacidade física/dependência como razão principal para o pedido do serviço $(\mathrm{n}=23 ; \%=52,3)$; no entanto, estes valores acentuam-se quando se verifica a presença de cuidador $(\mathrm{n}=17 ; \%=73,9)$. Dificuldades na confecção da alimentação constituem também uma das principais razões que justificam o pedido do serviço $(n=12 ; \%=27,3)$. Verifica-se, contudo, que este factor predomina nos receptores que não têm presença de cuidador $(\mathrm{n}=10 ; \%=83,3)$. 
Tabela 1 - Caracterização sócio-demográfica dos utentes de SAD. Portugal, 2008.

\begin{tabular}{|c|c|c|c|c|c|c|}
\hline \multirow[t]{2}{*}{ Características sócio-demográfica } & \multicolumn{2}{|c|}{ Total } & \multicolumn{2}{|c|}{$\begin{array}{l}\text { Presença de } \\
\text { cuidador }\end{array}$} & \multicolumn{2}{|c|}{ Ausência de cuidador } \\
\hline & $\mathrm{n}$ & $\%$ & $\mathrm{n}$ & $\%$ & $\mathrm{n}$ & $\%$ \\
\hline \multicolumn{7}{|l|}{ Sexo } \\
\hline $\begin{array}{l}\text { Masculino } \\
\text { Feminino }\end{array}$ & 15 & $31.3 \%$ & 6 & $40.0 \%$ & 9 & $60.0 \%$ \\
\hline Idade X (DP) & \multicolumn{2}{|c|}{$78.8(9.8)$} & \multicolumn{2}{|c|}{$80.6(10.6)$} & \multicolumn{2}{|c|}{$76.7(8.2)$} \\
\hline \multicolumn{7}{|l|}{ Estado Civil } \\
\hline Solteiro & 8 & $16.7 \%$ & 4 & $50.0 \%$ & 4 & $50.0 \%$ \\
\hline Casado & 18 & $37.5 \%$ & 10 & $55.6 \%$ & 8 & $44.4 \%$ \\
\hline Viúvo & 17 & $35.4 \%$ & 9 & $52.9 \%$ & 8 & $47.1 \%$ \\
\hline Divórcio & 5 & $10.4 \%$ & 2 & $40.0 \%$ & 3 & $60.0 \%$ \\
\hline \multicolumn{7}{|l|}{ Habilitações literarias } \\
\hline Escola primária incompleta ${ }^{1}$ & 30 & $62.5 \%$ & 16 & $53.3 \%$ & 14 & $46.7 \%$ \\
\hline Escola Primária $^{2}$ & 15 & $31.3 \%$ & 7 & $46.7 \%$ & 8 & $53.3 \%$ \\
\hline Ensino preparatório ${ }^{3}$ & 1 & $2.1 \%$ & 1 & $100 \%$ & 0 & $0.0 \%$ \\
\hline Curso de formação ${ }^{4}$ & 1 & $2.1 \%$ & 0 & $0.0 \%$ & 1 & $100 \%$ \\
\hline Curso Universitário ${ }^{5}$ & 1 & $2.1 \%$ & 1 & $100 \%$ & 0 & $0.0 \%$ \\
\hline
\end{tabular}

${ }^{1}$ Menos que 4 anos; ${ }^{2} 4$ anos de escolaridade; ${ }^{3} 6$ anos de escolaridade; ${ }^{4}$ mais do que 12 anos de escolaridade; ${ }^{5}$ Mais do que 12 anos de escolaridade.

Instrumentos - Protocolo de Serviço de

Apoio Domiciliário

Com base nos diferentes modelos de avaliação de serviços de cuidado destinados a idosos, ${ }^{10}$ foi criado um protocolo estruturado denominado Protocolo de Serviço de Apoio Domiciliário (ProSAD). ${ }^{11}$ Esse protocolo inclui uma compilação de itens relativos ao $\mathrm{SAD}$ e avalia suas diferentes dimensões.

Desse instrumento, foram analisados apenas os itens de avaliação da intensidade dos serviços prestados pelo SAD (variáveis dependentes) e de variáveis associadas a essa intensidade (variáveis independentes). Neste sentido, foram analisadas as variáveis de tempo diário disponibilizado por visita e por tarefa de cuidado, periodicidade das visitas e tempo de vinculação ao SAD.

\section{RESULTADOS}

Os resultados apresentam, em primeiro lugar, a análise da periodicidade das tarefas de cuidado prestadas pelo $\mathrm{SAD}$, e em segundo, a análise do tempo despendido. As tarefas de cuidado foram analisadas separadamente em relação a presença ou ausência de cuidador informal, com o objectivo de identificar a relação entre o serviço formal do SAD e o cuidado informal.

\section{Análise da periodicidade}

Da análise da periodicidade da prestação de serviços para cada utente (Tabela 2), concluiu-se que em termos de $\operatorname{AVD}(B)$, o serviço de alimentação, acompanhamento das refeições e mobilizações apresentam a moda mais elevada, sendo disponibilizados todos os dias. Por outro lado, os serviços de higiene íntima, levantar e vestir 
registaram uma média de frequência de segundafeira a sábado, e o banho completo é apenas fornecido uma vez por semana. Relativamente à análise em função da presença/ausência de cuidador, não se observam diferenças, com excepção do levantar e mobilizações, onde se observa maior frequência nos receptores de cuidados que têm cuidador.

Tabela 2-Periodicidade dos serviços em termos de AVD(B) e ADV(I). Portugal, 2008.

\begin{tabular}{|c|c|c|c|}
\hline & \multicolumn{3}{|c|}{ Periodicidade (moda) } \\
\hline & Total & $\begin{array}{l}\text { Presença } \\
\text { Cuidador }\end{array}$ & Ausência de Cuidador \\
\hline \multicolumn{4}{|l|}{$\begin{array}{l}\text { Serviços prestados em termos } \\
\text { de } \operatorname{AVD}(B)\end{array}$} \\
\hline Alimentação & Diária & Diária & Diária \\
\hline $\begin{array}{l}\text { Acompanhamento das } \\
\text { refeições }\end{array}$ & Diária & Diária & Diária \\
\hline Banho Completo & 1 dia na semana & 1 dia na semana & 1 dia na semana \\
\hline Higiene Íntima & Segunda a Sáb. & Diária & $\begin{array}{l}\text { Segunda a Sáb./1dia na } \\
\text { semana }\end{array}$ \\
\hline Levantar & Segunda a Sáb. & Segunda a Sáb. & a \\
\hline Mobilizações & Diária & Diária & a \\
\hline Vestir & Segunda a Sáb. & Segunda a Sáb. & Segunda a Sáb. \\
\hline \multicolumn{4}{|l|}{$\begin{array}{l}\text { Serviços prestados em termos } \\
\text { de AVD(I) }\end{array}$} \\
\hline Limpeza da Habitação & Segunda a Sáb. & Segunda a Sáb. & 1 dia na semana \\
\hline Tratamento de Roupas & 1 dia na semana & 1 dia na semana & 1 dia na semana \\
\hline Apoio na medicação & Diária & Diária & Diária \\
\hline Aquisição bens no exterior & $\begin{array}{l}1 \text { dia na semana/ } \\
\text { sempre que necessário }\end{array}$ & 1dia na semana/ & 1dia na semana/ \\
\hline \multicolumn{4}{|l|}{ Pequenos recados } \\
\hline Actividades de animação & 1 dia na semana & $\begin{array}{l}\text { Sempre que } \\
\text { necessário }\end{array}$ & $\begin{array}{l}\text { Sempre necessário } \\
1 \text { dia na semana }\end{array}$ \\
\hline \multicolumn{4}{|l|}{ Outras tarefas } \\
\hline & Sempre que necessário & $\begin{array}{l}\text { Sempre que } \\
\text { necessário }\end{array}$ & Sempre que necessário \\
\hline
\end{tabular}

a Ausência de prestação do serviço. 
Relativamente aos serviços associados às AVD(I), os serviços de limpeza da habitação, tratamento de roupas e apoio na medicação apresentam periodicidade muito frequente com uma moda que varia entre a diária e segundafeira a sábado (6 dias por semana). Os serviços de aquisição de bens no exterior e pequenos recados apresentam periodicidade menos frequente, entre um dia na semana, ou sempre que necessário. As actividades de animação sóciocultural têm periodicidade apenas pontual. Relativamente à presença/ausência de cuidador, os resultados não parecem evidenciar diferenças, com excepção do serviço de limpeza da habitação, que regista uma periodicidade mais elevada na presença de cuidador (segunda-feira a sábado), sendo que este resultado poderá ser justificado pelo facto de os utentes com cuidador tenham, possivelmente, menor capacidade funcional.
Análise do tempo diário total e por tarefa de cuidado

Apenas foi realizada a análise do tempo despendido (em minutos) em termos de $\operatorname{AVD}(\mathrm{B})$, relativamente ao tempo despendido nas AVD(I), não se procedeu ao seu cálculo, atendendo ao carácter muito pontual ou inexistente de que se reveste a prestação de serviços a este nível.

Verificou-se um tempo (em minutos) reduzido em termos de $\mathrm{AVD}(\mathrm{B})$ (Tabela 3). As tarefas associadas à higiene pessoal do utente são as que implicam uma maior utilização de tempo, designadamente o banho completo $(\mathrm{Mdn}=30 \mathrm{~min}$.; Min.=15 | Máx.=120) e a higiene íntima ( $\mathrm{Mdn}=$ 15,0 min.; Min.=10 | Máx= 30). A distribuição da alimentação utiliza também um tempo curto $(\mathrm{Mdn}=5,0$ min.; Min. =5 | Máx.=15), sendo que o acompanhamento das refeições é ainda mais reduzido $(\mathrm{Mdn}=10 \mathrm{~min} . ; \mathrm{Min} .=10 \mid$ Máx.=10).

Tabela 3 - Tempo despendido nas AVD(B). Portugal, 2008.

\begin{tabular}{lcccccc}
\hline \multirow{2}{*}{$\begin{array}{l}\text { Tempo Despendido } \\
\text { (em minutos) }\end{array}$} & \multicolumn{2}{c}{ Total } & \multicolumn{2}{c}{$\begin{array}{c}\text { Presença } \\
\text { Cuidador }\end{array}$} & \multicolumn{2}{c}{ Ausência de Cuidador } \\
\cline { 2 - 7 } & Mdn & $\begin{array}{c}\text { (Min- } \\
\text { Max) }\end{array}$ & Mdn & $\begin{array}{c}\text { (Min- } \\
\text { Max) }\end{array}$ & Mdn & $\begin{array}{c}\text { (Min- } \\
\text { Max) }\end{array}$ \\
\cline { 2 - 7 } & & & & & & \\
Alimentação & $5,0(\mathrm{n}=34)$ & $(5-15)$ & $15(\mathrm{n}=15)$ & $(5-15)$ & $5(\mathrm{n}=19)$ & $(5-15)$ \\
Acomp. das refeições & $10,0(\mathrm{n}=3)$ & $(10-10)$ & $10(\mathrm{n}=3)$ & $(10-10)$ & - & - \\
Banho Completo & $30,0(\mathrm{n}=21)$ & $(15-120)$ & $30(\mathrm{n}=15)$ & $(15-120)$ & $30(\mathrm{n}=6)$ & $(15-60)$ \\
Higiene Íntima & $15,0(\mathrm{n}=12)$ & $(10-30)$ & $15(\mathrm{n}=11)$ & $(10-30)$ & $15(\mathrm{n}=1)$ & $(15-15)$ \\
Levantar & $5,00(\mathrm{n}=1)$ & $(5-5)$ & $5(\mathrm{n}=1)$ & $(5-5)$ & - & - \\
Mobilizações & $7,5(\mathrm{n}=4)$ & $(5-15)$ & $7,5(\mathrm{n}=4)$ & $(5-15)$ & - & - \\
Vestir & $5(\mathrm{n}=14)$ & $(5-5)$ & $5(\mathrm{n}=13)$ & $(5-5)$ & $5(\mathrm{n}=1)$ & $(5-5)$ \\
\hline
\end{tabular}


Da análise dos resultados em função da presença/ausência de cuidador, constatou-se a mesma tendência que os resultados globais, apesar de existirem algumas diferenças. $\mathrm{O}$ banho completo continua a ser a tarefa que utiliza mais tempo, sobretudo nos receptores de cuidados que têm cuidador $(\mathrm{Mdn}=30 \mathrm{~min}$.; $\mathrm{Min} .=15$ Máx.=120), seguido da higiene íntima $(\mathrm{Mdn}=15$ min.; Min.=10 | Máx.=30).

A média do tempo despendido diariamente por utente é de $20 \mathrm{~min}$. (Mínimo = $5 \mathrm{~min}$. Máximo $=125 \mathrm{~min}$.).

Pelo exposto, conclui-se que o SAD em Portugal dedica ao utente uma média de 2,4 horas por semana $(\mathrm{DP}=2,3)$, tempo inferior ao que é disponibilizado em Espanha (4 horas por semana). ${ }^{12}$

\section{DISCUSSÃO}

Em Portugal, a periodicidade e o tempo afecto à prestação de serviços de $\mathrm{SAD}$ são bastante reduzidos, em comparação dados precedentes de países nórdicos. ${ }^{13}$ Estudos concluíram que a intensidade de serviços de qualidade inerentes ao SAD corresponde a três visitas semanais, com duração de uma hora e trinta minutos; no caso de idosos com maiores necessidades, são realizadas cinco visitas semanais, com duração superior a quatro horas. ${ }^{14}$

Os resultados obtidos em Portugal indicam uma afectação de tempo diário reduzida, sendo que os poucos serviços prestados incidem nas $\operatorname{AVD}(\mathrm{B})$ (alimentação e higiene pessoal), às quais se atribui um tempo limitado. $O$ banho completo é a tarefa à qual se afecta mais tempo, apesar de sua periodicidade média ser de um dia por semana, seguida da alimentação, que na maior parte dos casos apenas se limita à sua distribuição diária, precedida pela higiene íntima.

Os utentes que dispõem de cuidador principal são os que utilizam mais tempo de SAD. A utilização de tempo de SAD, com estes receptores de cuidados, incide sobretudo nas tarefas associadas às $\operatorname{AVD}(\mathrm{B})$, especificamente no acompanhamento das refeições, no banho completo e na higiene íntima. Tal resultado pode ser facilmente justificado pela maior necessidade de recurso aos serviços, dado que possivelmente se tratem de utentes que mais dependentes funcionalmente, pelo que necessitam de contar com a presença do cuidador principal.

Nestes casos, está implícito um modelo de utilização de SAD em termos de complementaridade, ${ }^{15}$ na qual se defende que, apesar de o utente poder contar com a colaboração do cuidador principal porque está dependente, o SAD surge como um complemento na prestação dos cuidados ao cuidador principal, e não como seu substituto.

Foi também possível concluir que nem todas as instituições disponibilizam seus serviços durante os sete dias da semana. O horário de funcionamento do $S A D$ poderá, assim, constituir também um dos factores determinantes para o pouco tempo disponibilizado. Ao contrário do que se verifica em Portugal, a abrangência de horário de funcionamento do SAD durante as 24 horas, em alguns países europeus, ${ }^{16}$ é uma realidade, sendo geralmente utilizada em combinação com serviços de telealarme.

\section{CONCLUSÃO}

A imputação de tempo a ser utilizado para cada utente deve ter por base critérios objectivos, com base no estado funcional e na rede de suporte social do utente. ${ }^{7}$ Os dados agora obtidos indicam que podem não existir critérios objectivos para a imputação do tempo de trabalho, cabendo possivelmente a cada responsável da instituição promotora de $\mathrm{SAD}$ a avaliação da situação concreta e a planificação da intervenção, sem recurso a nenhum critério previamente estabelecido.

Ainda relativamente ao tempo afectado, é importante destacar o horário de funcionamento do $\mathrm{SAD}$, isto é, o tempo de cobertura diário disponibilizado. Verificou-se que nenhuma das instituições disponibiliza a prestação do serviço 
para além das 19 horas, não assegurando, deste modo, qualquer apoio nocturno.

De qualquer forma, a intensidade de cuidado prestado pelo SAD é sempre muito reduzida, inclusive quando realizamos uma análise dos valores máximos de tempo obtidos. O tempo diário de $\mathrm{SAD}$ máximo de um utente foi de 125 min., o que exemplifica claramente a baixa intensidade de cuidado de SAD em Portugal.

Os dados apresentados parecem ser confiáveis, sobretudo devido ao facto de os mesmos terem sido recolhidos por uma amostragem aleatória. Não obstante, a maior dificuldade consiste no

\section{REFERÊNCIAS}

1. Fortinsky R, Madigan E. Home Care Advances. New York: Springer Publishing Company; 2000. p. 137-62.

2. Adams C, Defrates D, Travis K. Intensity of Care by Discipline for Selected Home Health Diagnoses. Home Care Provid 2000; 5 (6): 208-12.

3. Ranci C, Pavolini E. New Trends of Long-Term Care Policy in Western Europe. In: Encontro anual da American Political Science

Association. Chicago. (2006) . [acesso 2008 Mar.

12] Disponível em: URL: http://

www.allacademic.com/meta/

p208924_index.html.

4. Instituto de Migraciones y Servicios Sociales (IMSERSO). Calidad y Dependência: Grados de dependência y Necessidad de Servicios. Madrid: Ministerio de Trabajo y Asuntos Sociales; 2007.

5. Martin J, Neves R, Pires C, Portugal J.

Estatísticas de Equipamentos Sociais de Apoio à Terceira Idade em Portugal. Unidade de Investigação e Formação sobre Adultos e Idosos (UnIFai), 2006. Porto; 2006. p. 7-10.

6. CNIS. Protocolo de Cooperação de 2006 celebrado entre o Ministério do Trabalho e da Solidariedade Social e a Confederação Nacional das Instituições de Solidariedade. (2006). [acesso 2007 ago. 25]. Disponível em URL: www.solidariedade.pt/sartigo/index.

7. Instituto de Migraciones y Servicios Sociales (IMSERSO). Evolución y Extensión del Servicio de Ayuda a Domicilio en España. Madrid: Ministerio de Trabajo y Asuntos Sociales; 1998. significado dos dados do que pode ser considerado como de "baixa intensidade", isto é, a ausência de tempos standard considerados óptimos para a prestação de cuidados em SAD.

Esta linha de investigação seria consideravelmente beneficiada com a existência de estudos comparativos internacionais. Este tipo de estudos permitiria a análise do tempo despendido entre os diferentes países, mas controlando sempre o grau de dependência do utente, a disponibilidade familiar para o cuidado, assim como algumas características do sistema de cuidados, nomeadamente o financiamento.

8. Portugal. Ministério do Trabalho e da Segurança Social. Carta Social - Rede de Serviços e Equipamentos. Lisboa: MTSS. (2006) [acesso 2008 jan. 07] Disponível em: URL: http:// www.cartasocial.pt/pdf/csocial2006.pdf.

9. INE - Instituto Nacional de Estatística (Portugal). Página Oficial do Instituto Nacional de Estatística. Portugal: INE. (2006). [acesso 2006, dez. 01] Disponível em: URL: http:// www.INE.pt.

10. Doyle C. How to assess the quality of long term care, 1991. Autrália: Centre for Health Program Evaluation. (1991) [acesso 2008 jul. 5]. Disponível em: URL: http://www.buseco.monash.edu.au/ centres/che/pubs/wp14.pdf.

11. Martin I, Oliveira M, Cunha C. Protocolo de Avaliação do Serviço de Apoio Domiciliário (ProtSAD v.2), 2007. Porto: UnIFai. [acesso 2007 Mar. 14] Disponível em: URL: http:// www.unifai.eu/files/active/1/ DossierTecnico_ProSAD.pdf

12. Espanha. Ministerio de Trabajo y Asuntos Sociales. Libro Blanco de la Dependencia. Secretaria de Estado de Servicios Sociales Familias y Discapacidad; 2004.

13. Nordic Social-Statistical Commitee. Social Protection in the Nordic Countries 2000. Scope, expenditure and financing. Copenhagen: Nordic Social-Statistical Committee; 2002.

14. Struyk R, Alexandrova A, Belyakov I, Chaging K. Client Satisfaction with Home Care Services in Rural Russia. J Aging Soc Policy 2006, 18 (1): 87-105. 
15. Martin I, Gonçalves D, Paúl C, Pinto C.

Políticas Sociais para a Terceira Idade em

Portugal. In: Osório R. Pessoas Idosas: Contexto

Social e Intervenção Educativa. Lisboa; 2006.
16. Seutin, R. Planificación et Programmation des Services D'Aide à Domicile. In Seminário Europeu - Apoio Domiciliário. Lisboa: Direcção Geral de Acção Social; 1999. p. 44-50.

Recebido: 25/11/2008

Revisado: 12/10/2009

Aprovado: 09/4/2010 
